Please do not remove this page

RMIT

UNIVERSITY

\title{
Elastomeric silicone substrates for terahertz fishnet metamaterials
}

Khodasevych, Iryna; Shah, Charan; Sriram, Sharath; Bhaskaran, Madhu; Withayachumnankul, W.; Ung, B; Lin, $\mathrm{H}$

https://researchrepository.rmit.edu.au/esploro/outputs/9921858422201341/filesAndLinks?institution=61RMIT_INST\&index=null

Khodasevych, I., Shah, C., Sriram, S., Bhaskaran, M., Withayachumnankul, W., Ung, B., Lin, H., Rowe, W., Abbott, D., \& Mitchell, A. (2012). Elastomeric silicone substrates for terahertz fishnet metamaterials. Applied Physics Letters, 100(6), 061101-1-061101-061103. https://doi.org/10.1063/1.3665180 Document Version: Accepted Manuscript

Published Version: https://doi.org/10.1063/1.3665180

Repository homepage: https://researchrepository.rmit.edu.au (C) 2012 American Institute of Physics

Downloaded On 2023/04/26 23:07:47 +1000 
Thank you for downloading this document from the RMIT Research Repository.

The RMIT Research Repository is an open access database showcasing the research outputs of RMIT University researchers.

RMIT Research Repository: http://researchbank.rmit.edu.au/

\section{Citation:}

Khodasevych, I, Shah, C, Sriram, S, Bhaskaran, M, Withayachumnankul, W, Ung, B, Lin, H, Rowe, W, Abbott, D and Mitchell, A 2012, 'Elastomeric silicone substrates for terahertz fishnet metamaterials', Applied Physics Letters, vol. 100, no. 6, 061101, pp. 1-3.

See this record in the RMIT Research Repository at:

http://researchbank.rmit.edu.au/view/rmit:14411

Version: Accepted Manuscript

Copyright Statement: (c) 2012 American Institute of Physics

Link to Published Version:

http://dx.doi.org/10.1063/1.3665180 


\title{
Elastomeric silicone substrates for terahertz fishnet metamaterials
}

I. E. Khodasevych, ${ }^{1}$ C. M. Shah, ${ }^{1}$ S. Sriram, ${ }^{1, a)}$ M. Bhaskaran, ${ }^{1}$ W. Withayachumnankul, ${ }^{2,3}$

B. S. Y. Ung, ${ }^{2}$ H. Lin, ${ }^{2}$ W. S. T. Rowe, ${ }^{1}$ D. Abbott,${ }^{2}$ and A. Mitchell ${ }^{1,4}$

${ }^{1}$ Microplatforms Research Group, School of Electrical and Computer Engineering, RMIT University, GPO Box 2476, Melbourne, VIC 3001, Australia

${ }^{2}$ School of Electrical \& Electronic Engineering, The University of Adelaide, Adelaide, SA 5005, Australia

${ }^{3}$ Faculty of Engineering, King Mongkut's Institute of Technology, Ladkrabang, Bangkok 10240, Thailand

${ }^{4}$ ARC Centre for Ultrahigh bandwidth Devices for Optical Systems (CUDOS), School of Electrical and Computer Engineering, RMIT University, GPO Box 2476, Melbourne, VIC 3001, Australia

(Received

\begin{abstract}
In this work, we characterize the electromagnetic properties of polydimethylsiloxane (PDMS) and use this as a free-standing substrate for the realization of flexible fishnet metamaterials at terahertz (THz) frequencies. Across the 0.2 to $2.5 \mathrm{THz}$ band, the refractive index and absorption coefficient of PDMS are estimated as 1.55 and $0-22 \mathrm{~cm}^{-1}$, respectively. Electromagnetic modeling, multi-layer flexible electronics microfabrication, and terahertz time-domain spectroscopy are used in the design, fabrication, and characterization of the metamaterials, respectively. The properties of PDMS adds a degree of freedom to terahertz metamaterials, with the potential for tuning by elastic deformation or integrated microfluidics.
\end{abstract}

\section{KEYWORDS}

flexible devices, terahertz metamaterials, fishnet, PDMS 
Terahertz $(\mathrm{THz})$ or T-ray radiation is attracting much attention in recent years with the development of biomedical imaging, security screening, manufacturing quality control, and sensors. ${ }^{1-3}$ Terahertz applications are further augmented by the progress made in the field of metamaterials, since natural materials only exhibit weak electric and magnetic responses at terahertz frequencies. ${ }^{4}$ Metamaterials, unlike natural materials, can be artificially tailored to exhibit strong resonant behavior at terahertz frequencies.

Since the first demonstration of metamaterials for microwave frequencies, ${ }^{5}$ the majority of demonstrated metamaterials have been fabricated on rigid substrates. However, there is demand for flexible metamaterials that can be wrapped around objects for cloaking applications ${ }^{6}$ or attached to curved surfaces to serve as strain sensors. ${ }^{3}$ In addressing this need, flexible split-ring structures on polyimide $^{6}$ and parylene ${ }^{7}$ have recently been reported, as well as crosses embedded in benzocyclobutene. ${ }^{8}$

In this work, a fishnet metamaterial structure is realized at terahertz frequencies by using flexible material as a substrate. The fishnet structure is generally comprised of two metal layers, which is well served by planar processing. Moreover, in the terahertz frequency regime, fishnet unit cell dimensions are in the micro-scale, and compatible with standard semiconductor fabrication techniques. Unlike planar arrays of split-ring resonators, fishnets are capable of exhibiting negative permittivity and negative permeability simultaneously, achieving the negative index of refraction required for a number of applications such as perfect lenses. ${ }^{9}$ Fishnet structures also allow realization of polarization insensitive designs. $^{10}$

While polyimide $^{6}$ and parylene ${ }^{7}$ are suitable as flexible substrates, in this work we have chosen the elastomeric silicone polymer - polydimethylsiloxane (PDMS), which is a low cost highly elastic material that is already widely used in microfluidics and nanofabrication. ${ }^{11-13}$ Also, the low surface energy of PDMS allows conformal adhesion to curved surfaces. 
As a silicone polymer, PDMS is known to have stable dielectric properties and low transmission loss. ${ }^{14}$ This behavior is verified in the terahertz regime using a free-space coupled terahertz time-domain spectroscopy (THz-TDS) system, equipped with a photoconductive antenna for T-ray generation and electro-optical detection. The pumping laser was a mode-locked Ti:sapphire laser with a central wavelength of $800 \mathrm{~nm}$, a pulse duration of $<100 \mathrm{fs}$, and a repetition rate of $80 \mathrm{MHz}$. The system generated pulsed T-ray radiation spanning 0.05 to $2.8 \mathrm{THz}$, with a maximum dynamic range of $30 \mathrm{~dB}$. The collimated beam diameter was approximately $10 \mathrm{~mm}$.

The PDMS samples under test were 375 and $960 \mu \mathrm{m}$ thick sheets. Reference measurements were conducted at room temperature. The index of refraction and the absorption coefficient for both thicknesses were determined, with similar characteristics obtained. The refractive index and absorption coefficient for the $960 \mu \mathrm{m}$ thick PDMS sheet in the terahertz region are shown in Fig. 1(a) and 1(b), respectively. The absorption coefficient of $20 \mathrm{~cm}^{-1}$ at $1.0 \mathrm{THz}$ compares well with that of $30 \mathrm{~cm}^{-1}$ for polyimide. ${ }^{6}$ Using these results, the dielectric constant and loss tangent of PDMS were estimated as 2.35 and $0.02-0.04$, respectively, across the 0.2 to $2.5 \mathrm{THz}$ band. This relatively low absorption of PDMS is significant to a reduction of loss in metamaterials. ${ }^{15}$

The unit cell of the proposed fishnet structure ${ }^{10,16}$ is shown in Fig. 2. The structure consists of patterned metallic layers embedded in PDMS. This fishnet is independent of polarization of the incident wave due to its symmetric square structure. Using these dielectric properties, fishnet structures were designed using Ansoft HFSS software (based on the full-wave finite element method). The structure is simulated as both periodic and infinite in the $x-y$ plane. The incident wave is normal to the plane of the structure, and the electric and the magnetic fields are parallel to $y$-axis and $x$-axis, respectively.

The simulated performance of the designed fishnet is presented as Supplemental Material (Fig. S1). ${ }^{17}$ The fishnet exhibits a magnetic resonance at $2.1 \mathrm{THz}$ due to antiparallel currents induced in the first and second layers of the structure by the magnetic field passing between the metal layers. The 
currents form an inductive-capacitive $L C$ loop within each unit cell, with the spacer between the layers acting as a capacitor and metal slabs acting as inductors. ${ }^{18}$

The effective permittivity and permeability of the fishnet, presented in Fig. 3, were extracted from the simulation results. ${ }^{19}$ The sample transmission and reflection coefficients were de-embedded (based on Fig. 2) to compensate for the phase shift in the outer PDMS layers. The structure clearly shows a negative permittivity below $1.7 \mathrm{THz}$ [Fig. 3(a)] and a negative permeability at $2.1 \mathrm{THz}$ [Fig. 3(b)]. These engineered negative properties are clear evidence of the metamaterial effect of the sub-wavelength resonator features. However, in our realized structure, these electric and magnetic properties are not found at the same frequency as would be required for a negative refractive index metamaterial. Such a structure could be achieved by optimizing the unit cell to account for the plasma frequency of the wire grid.

The behavior of the fishnet structure is strongly dependent on the direction of flow of its surface currents. The surface current in the top and bottom metal layers of the fishnet at $2.1 \mathrm{THz}$ is presented in Fig. 4(a) and 4(b), respectively. This shows that the current flows in opposite directions, as is expected at the frequency of the magnetic resonance and hence the negative permeability. ${ }^{19}$ The current distribution at electric resonance is presented in the Supplemental Material as Fig. S2. ${ }^{17}$ A video depicting the evolution of surface currents to illustrate the physical model of the terahertz fishnet is also presented as Supplemental Material (Video S1). ${ }^{17}$

The fishnet structures are fabricated for experimental validation exploiting standard microfabrication techniques that are adapted to flexible, elastomeric device fabrication. ${ }^{20}$ The fishnets have a total of 5 layers, with alternating layers of PDMS (3 layers) and patterned metal (2 layers). PDMS is spin coated on to a silicon wafer ${ }^{19}$ to obtain a thickness of $250 \mu \mathrm{m}$. Metal layers are deposited on the PDMS coated wafer using electron beam evaporation at room temperature. Alternating layers of chromium $(20 \mathrm{~nm})$ and gold $(200 \mathrm{~nm})$ are deposited with three layers of chromium encapsulating two 
layers of gold. The alternating layers are to minimize thin film stress, and also to have chromium as the outer layers to improve adhesion to PDMS, resulting in a $460 \mathrm{~nm}$ thick metal layer. The metal layers are patterned using standard photolithography techniques. Full details on the fabrication and patterning processes are provided as Supplemental Material. A thin layer of PDMS $(10 \mu \mathrm{m})$ is spin-coated on the first metal fishnet layer to serve as the dielectric separating the two fishnet layers. Following the curing of this layer, the multi-layer metallization and etching process is repeated to define the second fishnet layer, with an alignment process during photolithography. The fabrication process is completed by spin coating and curing a $250 \mu \mathrm{m}$ thick PDMS layer. The resulting 5-layered symmetric structure is peeled off from the supporting silicon substrate to realize a flexible fishnet metamaterial [Fig. 5(a)].

The elastomeric property of PDMS that this work aims to capitalize on, poses a challenge in the alignment of layers during the patterning of the second fishnet layer with respect to the first. The contact lithography process occurs with a wedge error correction process, meaning one edge of the wafer comes into contact with the photolithography mask first, before the rest gradually comes in contact. This typically results in a 2-5 $\mu \mathrm{m}$ misalignment error in the multilayer elastomer samples. Figure 5(b) shows a fabricated fishnet structure with $3 \mu \mathrm{m}$ misalignment in both $x$ and $y$ directions. The influence of this misalignment on the expected terahertz spectra is determined by HFSS modeling with the results presented $^{17}$ in Fig. S3. The misalignment causes a slight splitting of both the stop band and also the ensuing pass band. Despite this slight splitting, the structure shows good tolerance to misalignment, in that the magnitude and position of the transmission band remain almost the same.

Figure 6 compares the simulated and measured results for transmission through the fishnet structure. Good agreement between theoretical and experimental results is observed. Resonant dips are evident at $2.0 \mathrm{THz}$ and $2.1 \mathrm{THz}$ as predicted by simulations of a slightly misaligned fishnet. Splitting in the high transmission area after resonance is also evident in both the experimental measurements and theoretical predictions. The lowered transmission below $1.65 \mathrm{THz}$ is caused by the negative permittivity achieved by the metamaterial. The simulated results provide a best fit to the experimental results for a 
loss tangent of 0.02, validating the low loss characteristics of the elastomeric PDMS substrates. The weak oscillations observed in the measurement are caused by Fabry-Pérot reflections (discussed in Supplemental Material) ${ }^{17}$ and do not affect the performance of the fishnet.

In conclusion, we have demonstrated the fabrication and characterization of a fishnet metamaterial operating in the terahertz frequency range using the flexible elastomer PDMS for all dielectric layers. A strong magnetic resonance is observed. The fishnet embedded in thick PDMS outer layers shows high transmission values in the pass bands highlighting the low propagation loss of the PDMS material. The structure exhibits a high tolerance to layer misalignment, maintaining the approximate position and strength of the resonances with only slight splitting of the resonances observed as a result of layer misalignment. Our future work will explore the utilization of the microfluidic capabilities of PDMS to enable fluid infiltration for tuning and sensing applications. We will also explore the conformal and adhesive properties of PDMS to use this realized terahertz fishnet as an in situ remotely interrogated sensing platform.

\section{ACKNOWLEDGEMENTS}

SS, MB, and WW acknowledge Australian Post-Doctoral Fellowships from the Australian Research Council (ARC) through Discovery Projects DP110100262, DP1092717, and DP1095151, respectively. ARC funding through Discovery Projects DP0988673 and DP1097281 (DA) and the Centres of Excellence scheme (AM) are acknowlegded.

\section{NOTES}

${ }^{a)}$ Corresponding author. E-mail: sharath.sriram@gmail.com 


\section{REFERENCES}

1. M. Tonouchi, Nat. Photon. 1, 97 (2007).

2. W. Withayachumnankul, G. M. Png, X. Yin, S. Atakaramians, I. Jones, H. Lin, B. S. Y. Ung, J. Balakrishnan, B. W.-H. Ng, B. Ferguson, S. P. Mickan, B. M. Fischer, and D. Abbott, Proc. IEEE 95, 1528 (2007).

3. R. Melik, E. Unal, N. K. Perkgoz, C. Puttlitz, and H. V. Demir, Appl. Phys. Lett. 95, 181105 (2009).

4. W. Withayachumnankul and D. Abbott, IEEE Photon. J. 1, 99 (2009).

5. D. R. Smith, W. J. Padilla, D. C. Vier, S. C. Nemat-Nasser, and S. Schultz, Phys. Rev. Lett. 84, 4184 (2000).

6. H. Tao, A. C. Strikwerda, K. Fan, C. M. Bingham, W. J. Padilla, X. Zhang, and R. D. Averitt, J. Phys. D: Appl. Phys. 41, 232004 (2008).

7. X. Liu, S. MacNaughton, D. B. Shrekenhamer, H. Tao, S. Selvarasah, A. Totachawattana, R. D. Averitt, M. R. Dokmeci, S. Sonkusale, and W. J. Padilla, Appl. Phys. Lett. 96, 011906 (2010).

8. O. Paul, C. Imhof, B. Reinhard, R. Zengerle, and R. Beigang, Opt. Exp. 16, 6736-6744 (2008).

9. J. B. Pendry, Phys. Rev. Lett. 85, 18 (2000).

10. P. Ding, E. J. Liang, W. Q. Hu, L. Zhang, Q. Zhou, and Q. Z. Xue, Photon. Nanostruct.: Fundam. Appl. 7, 92100 (2009).

11. Y. Xia and G. M. Whitesides, Angew. Chem. Int. Ed. 37, 550 (1998).

12. J. N. Lee, C. Park, and G. M. Whitesides, Anal. Chem. 75, 6544 (2003).

13. J. A. Rogers and R. G. Nuzzo, Mater. Today 8, 50 (2005).

14. Sylgard 184 Silicone Elastomer Kit Product Data Sheet, Dow Corning Corporation.

15. R. B. Greegor, C. G. Parazzoli, K. Li, and M. H. Tanielian, Appl. Phys. Lett. 82, 2356 (2003).

16. S. Zhang, W. Fan, K. J. Malloy, and S. R. J. Brueck, Opt. Express 13, 4922 (2005).

17. See supplementary material at [URL will be inserted by AIP].

18. M. Kafesaki, I. Tsiapa, N. Katsarakis, T. Koschny, C. M. Soukoulis, and E. N. Economou, Phys. Rev. B 75, 235114 (2007).

19. D. R. Smith, S. Schultz, P. Markos, and C. M. Soukoulis, Phys. Rev. B 65, 195104 (2002).

20. C. M. Shah, S. Sriram, M. Bhaskaran, and A. Mitchell, Proc. 2010 Conf. Optoelectron. Microelectron. Mater. Dev. 187 (2010).

21. J. C. Lötters, W. Olthuis, P. H. Beltink, and P. Bergveld, J. Micromech. Microeng. 7, 145 (1997). 


\section{FIGURE CAPTIONS}

FIG. 1. Properties of a $960 \mu \mathrm{m}$ thick PDMS sheet measured in the terahertz region: (a) index of refraction and (b) absorption coefficient.

FIG. 2. Fishnet unit cell with the following parameters: cell dimensions $70 \times 70 \times 510 \mu \mathrm{m}^{3}, p=50 \mu \mathrm{m}, w=$ $5 \mu \mathrm{m}, t=10 \mu \mathrm{m}, d=250 \mu \mathrm{m}$ for both outer encapsulating PDMS layers.

FIG. 3. Effective permittivity and permeability of the double layer fishnet in PDMS are shown in (a) and (b), respectively.

FIG. 4. Distribution of the surface current of the (a) top and (b) bottom metal layer of the fishnet at the frequency of negative permeability $(\sim 2.1 \mathrm{THz})$. The opposite direction of current flow at magnetic resonance can be observed.

FIG. 5. (a) Photograph of a section of the microfabricated elastomeric fishnet structure. (b) Optical micrograph showing 9 fishnet unit cells, the void between the metallization appears as a cross, where a slight misalignment between the two metal layers can be observed. The dimensions of the fabricated structure are as presented in Fig. 2.

FIG. 6. Simulated and measured terahertz response of PDMS-based fishnet structures. Good agreement can be observed in transmission intensity and resonant dip position, including the expected stop band splitting due to the $3 \mu \mathrm{m}$ misalignment. 


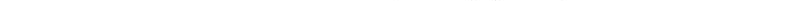




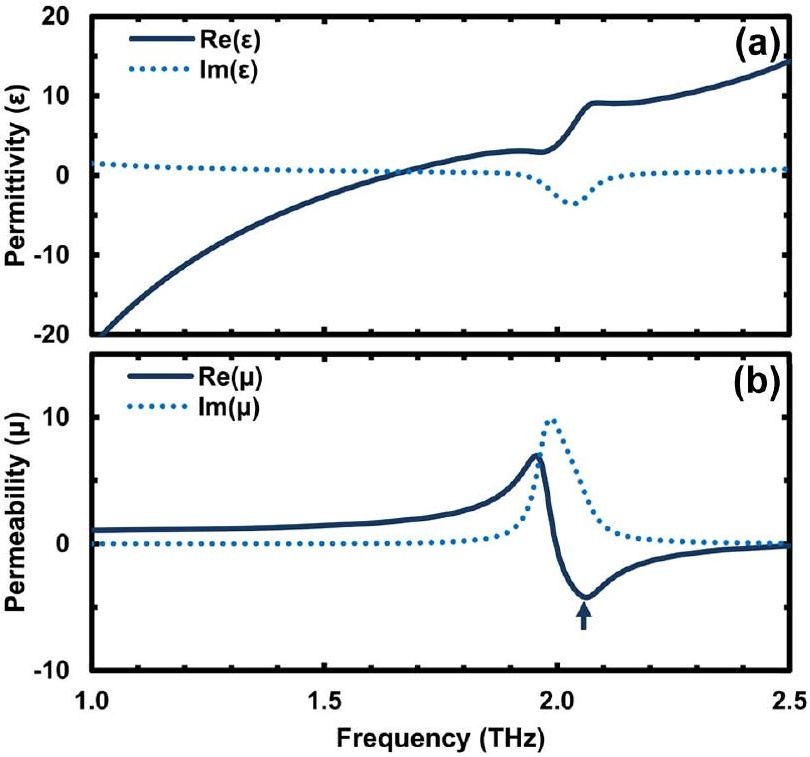




$$
10
$$




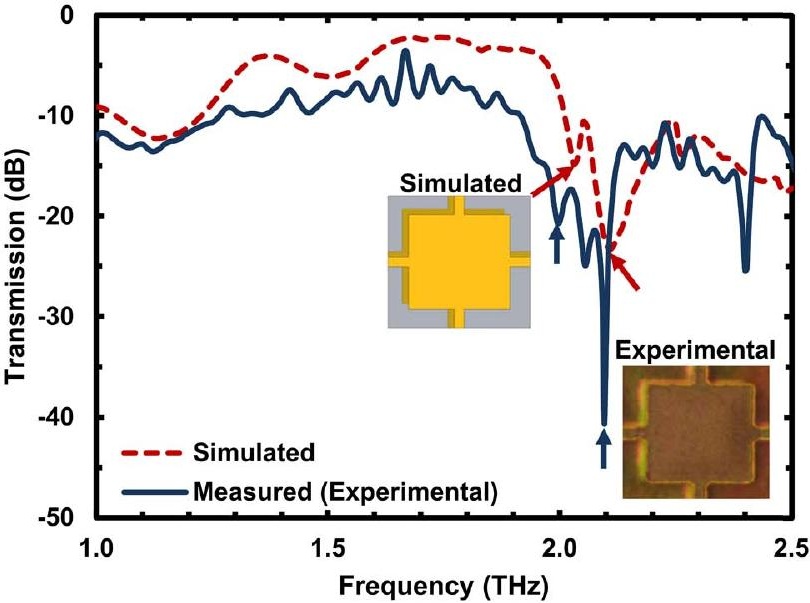

